\title{
Abstracts of the meeting of the Clinical Genetics Society held on 8, 9, and 10 November 1984 at the Royal Free Hospital, London
}

Genetic investigation of cleft lip with or without cleft palate. A three generation family study of $\mathbf{5 7 8}$ pedigrees M TOLAROVA

Institute of Experimental Medicine, Czechoslovak Academy of Sciences, Lidovych Milici 61, 12000 Prague 2, Czechoslovakia.

A family study of cleft lip with or without cleft palate was carried out on probands born in Bohemia between 1920 and 1955. The population of affected children was $4.80 \%$ $( \pm 0.75)$, of sibs $3.44 \%( \pm 0.57)$, and of grandchildren $2.01 \%( \pm 1 \cdot 16)$. The proportion of affected children increased with the clinical severity of the abnormality in the probands, $3.68 \%( \pm 0.72)$ for unilateral cleft lip against $10 \cdot 14 \%( \pm 2 \cdot 62)$ for bilateral cleft lip, and with the sex of the proband, $5 \cdot 35 \%( \pm 1 \cdot 30)$ for those of females and $4.47 \%( \pm 0.91)$ of males. The value for the heritability by Falconer's method is estimated for all the relatives: $h^{2}=0.7302( \pm 0.0445)$ for sibs and $h^{2}=0.8267( \pm 0.0442)$ for children. Based on these findings a four threshold model of $\mathrm{CL} \pm \mathrm{P}$ inheritance is suggested.

Four pregnancies with a male triploid fetus

K M LAURENCE

Department of Child Health, University of Wales College of Medicine, Heath Park, Cardiff.

In the 22 years between 1961 and 1983 only one triploid has been found; in nine months of 1984 there have been three cases. One of these four was a spontaneously aborted fetus with gross malformations, two were abnormal fetuses terminated in mid-trimester, and one had a spontaneous onset of labour at 33 weeks with the birth of an abnormal fetus who survived one hour. All the last three were associated with a sudden onset of severe toxaemia and a molar placenta, and two with grossly raised serum AFP but normal amniotic AFP levels. It is concluded that the presence of a male triploid fetus can be inferred from the sudden onset of toxaemia, the large molar placenta in the presence of a fetus, and a raised serum AFP.

Linkage analysis of the $X$ chromosome short arm leading to genetic counselling of the $X$ linked muscular dystrophies using multiple flanking DNA polymorphisms

D E WILCOX, N AFFARA, J R W YATES, AND M A FERGUSON-SMITH Duncan Guthrie Institute of Medical Genetics, Yorkhill, Glasgow.

Sixteen three generation families from the west of Scotland with Duchenne or Becker muscular dystrophy (DMD, BMD) were analysed using the $\mathrm{Xg}$ blood group and five cloned DNA sequences which recognise DNA polymorphisms on the short arm of the $\mathrm{X}$ chromosome (Xp). This generated useful linkage data between the disease loci and each of these markers. More importantly, 10 of these families were informative for at least two marker loci in addition to the disease locus and had third generation persons who were recombinants. These subjects allowed the loci to be ordered on Xp using the three point cross linkage. The proposed order based on both forms of linkage data is: centromere-L1.28-754-DMD/BMD-99/ $6-\mathrm{D} 2-782-\mathrm{Xg}$. These results conclusively map both DMD and BMD to the central area of Xp. Even though precise linkage distances between a probe and the disease loci may not be known, knowledge of its position can greatly aid genetic counselling when it is used with a marker on the other side of the disease locus. Flanking probes can predict the passage of the disease locus to the next generation and only an unlikely double recombination within the markers and on either side of the disease locus would affect the prediction.

Practical considerations in the application of linked DNA polymorphisms in families at risk for Duchenne muscular dystrophy

R F MUELler, B C C DAVISON, AND M VAN ALTAAN Department of Genetic Counselling, Addenbrooke's Hospital, Hills Road, Cambridge.

Family studies of the segregation of linked RFLPs may allow modification of the risk of a woman being a carrier for Duchenne muscular dystrophy. Review of the 40 DMD families seen by the Genetic Counselling Department in East Anglia showed that of the 40 families at risk 11 were not informative. In 12 of the potentially informative families, the potential consultands were below reproductive age so DNA studies are not indicated at present. Of these, only half are likely to be informative for the existing RFLPs and to enable one to modify the risk counselling. We suggest that before further contact is made with these families, particularly before blood is taken for DNA, they should be analysed in detail so that the samples are obtained in a systematic manner. In the first instance blood should only be taken from older affected males and from obligate or potential female heterozygotes to determine whether they are informative for the RFLPs before obtaining samples from other family members. 
Application of an intragenic genomic probe to genetic counselling for haemophillia $B$

J M CONNOR, A F PETTIGREW, I M HANN, C D FORBES, AND N AFFARA

Departments of Medical Genetics and Haematology, University of Glasgow, Glasgow.

A total ascertainment study showed 30 families with haemophilia B in the west of Scotland. In 12 of these families more than one person was affected and 26 living obligate carriers were identified and tested. Of these, $42 \%$ were heterozygous for a factor IX genomic probe. No recombination was observed. Definitive genetic counselling was possible for 14 of 42 females at risk; 11 could not be traced; in 10 the probe was not informative; and in seven paternal absence prevented interpretation. Marked linkage disequilibrium was apparent for this restriction fragment length polymorphism and haemophilia B in the west of Scotland.

Lethal osteogenesis imperfecta. Is the collagen gene deletion a normal variant rather than a causal mutation?

B SYKES AND D OGILVIE

Nuffield Department of Pathology, John Radcliffe Hospital, Headington, Oxford.

Four cases of perinatal lethal osteogenesis imperfecta have previously been described in which the probands were heterozygous for a deleted ' $\alpha_{1}$-like' collagen gene. It was proposed that this deletion was a contributory pathological mutation which could be used as a marker for antenatal diagnosis and, possibly, for termination of subsequent pregnancies. As three of four cases originated from India we examined a series of normal subjects from this and other populations. We found apparently similar deleted alleles occurring at a high frequency in Asian Indians $(0 \cdot 12, n=128)$ and West Indian Negroes $(0 \cdot 11, n=56)$ but very rarely in Caucasians $(0.003, n=678)$. These results favour our alternative explanation that the deletions described in these cases of osteogenesis imperfecta, and in a West Indian family with Ehlers-Danlos syndrome, are normal variants of the gene rather than causal mutations. We have localised the deletion to a segment of DNA just beyond the $3^{\prime}$ end of the gene (now identified as that encoding $\alpha_{1}$ (II) cartilage collagen). Closer examination has shown slight differences in the size of 'deleted' alleles. We consider that this segment of DNA is highly polymorphic. We speculate that, by analogy with the hypervariable regions flanking the $\alpha$ globin and insulin genes, it may consist of tandem repeats and that the polymorphism we observe could be due to unequal recombination with this segment.

Mitochondrial myopathy: a genetic study A E HARDING, R K H PETTY, AND J A MORGAN-HUGHES Institute of Neurology, Queen Square, London.

Sixty-one patients ( 29 males and 32 females) with histologically defined mitochondrial myopathy have been investi- gated at the National Hospital over the period 1969 to 1984 and over 40 of these have been reassessed in the last year. Three groups of cases could be identified clinically: a combination of chronic progressive external ophthalmoplegia with weakness of the limbs $(60 \%)$, fatigueable limb weakness alone $(15 \%)$, and those with clinical features (such as ataxia, dementia, pyramidal signs, involuntary movements, and seizures) predominantly or exclusively confined to the central nervous system $(25 \%)$. Nine index cases had relatives who were definitely affected with a similar disorder, and a more uncertain family history was obtained in a further six. Index cases with fatigueable limb weakness alone were more likely to have affected relatives than patients in the other two clinical groups. Overall, the recurrence rate was $3.6 \%$ for sibs and $8.7 \%$ for offspring of index cases; it was highest for sisters $(9.1 \%)$ and daughters $N$ $(15 \%)$ of female index cases. No consistent pattern of iv inheritance was apparent from the pedigree data, and $\vec{\omega}$ there were some findings which are incompatible with the hypothesis that mitochondrial myopathy is mitochondrially inherited.

Lethal X linked myotubular myopathy

H WILliams, G COLE, N THOMAS, C BROWN, AND M SARFARAZI University of Wales College of Medicine, Cardiff.

A three generation family with 10 affected pregnancies is described. Five successive affected pregnancies in one woman occurred and her sister's three pregnancies also only produced affected boys. The occurrence of affected males only in these two sisters is difficult to explain on the grounds of chance only. Affected pregnancies are characterised by reduced fetal movements and polyhydramnios, thought to be due to swallowing difficulties in utero. Affected infants die soon after birth from respiratory failure. A clinical and histopathological description is given. Attempts at carrier detection using biochemical tests and muscle biopsies have been inconclusive in the women at risk. Preliminary DNA studies in this family suggest that this $\mathrm{X}$ linked muscular disorder may be located on the short arm of the $\mathrm{X}$ chromosome.

Emery-Dreifuss muscular dystrophy

P K THOMAS AND R K H PETTY

Institute of Neurology, Queen Square, London.

Emery and Dreifuss ( $J$ Neurol Neurosurg Psychiatry 1966;29:338-42) described an unusual $X$ linked muscular dystrophy with an onset in childhood of proximal muscle weakness, contractures at the elbows, limitation of neck flexion, and an association with cardiomyopathy. These features are similar to those exhibited by further $\mathrm{X}$ linked families described by Rotthauwe et al (Humangenetik 1976;16:181-200) and Thomas et al (J Neurol Neurosurg Psychiatry 1972;35:208-15) but in which there was a scapuloperoneal distribution of muscle weakness. It has been suggested by Rowland et al (Ann Neurol 1979;5:1117) that both presentations are due to the same abnormal gene. This conclusion is supported by the present case, 일

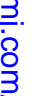
.

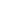
. 格

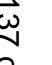
3

$$
\text { 言 }
$$

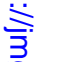

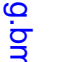

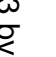
踾

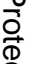

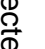
年

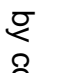


from a new $\mathrm{X}$ linked pedigree, which showed clinical features that were intermediate between the two syndromes. A muscle biopsy revealed myopathic changes of unusual type.

\section{High resolution cytogenetic analysis of chromosomal trans- locations associated with Duchenne muscular dystrophy in females \\ Y BOYD, $V$ J BUCKLE, AND J H EDWARDS \\ Genetics Laboratory, Biochemistry Department, University of Oxford, Oxford.}

The defective gene or genes which cause the sex linked Duchenne (DMD) and Becker (BMD) muscular dystrophies have been assigned to the chromosomal band Xp21 by the discovery of several girl patients carrying de novo translocations with breakpoints in this region. High resolution cytogenetic analysis was undertaken of lymphoblastoid cell lines established from eight patients in order to make a detailed study of those breakpoints. In four, and possibly five, of the eight cases an apparently simple reciprocal translocation was observed with the $X$ chromosome breakpoint in the subband Xp21.2. The sixth patient had a complex chromosomal rearrangement involving both a reciprocal translocation and an inversion between Xp21.2 and Xp11.4. These results confirm and extend previous observations. The simplest interpretation of the banding pattern observed in the final two cases is that a reciprocal translocation has occurred with an $X$ chromosome breakpoint in the band $\mathrm{Xp} 21 \cdot 1$, approximately $2000 \mathrm{~kb}$ from Xp21.2. However, alternative interpretations based on Xp21.2 breakpoints and complex rearrangements are plausible.

Prenatal diagnosis of cystic fibrosis using monoclonal antibodies specific for the isoenzymes of alkaline phosphatase

D J H BROCK AND $\mathrm{V}$ VAN HEYNINGEN

University Human Genetics Unit, and MRC Clinical and Population Cytogenetics Unit, Western General Hospital, Edinburgh.

We have tested three monoclonal antibodies, each with specificity for one of the major isoenzymes of alkaline phosphatase (ALP) in the prenatal diagnosis of cystic fibrosis. On a panel of stored amniotic fluids the direct immunoassay of intestinal ALP gave superior results to those achieved with enzymatic determination of $\gamma$ glutamyltranspeptidase, aminopeptidase $\mathbf{M}$, maltase, or amino acid inhibited ALP. Application of the immunoassay to 100 prospective high risk pregnancies has shown that both false positives and false negatives do occur. Estimation of the exact sensitivity and specificity has been complicated by difficulties in following up outcomes in the prospective series. However, it now seems possible to confirm diagnoses in abortus material by inspection of the fetus for 'meconium ileus' and by measuring proteins and enzymes in meconium samples. It has also become apparent that the optimal time for amniocentesis is after 17 weeks of pregnancy. It seems probable that if this form of prenatal diagnosis is applied to 1 in 4 risk pregnancies, a false negative rate of 10 to $15 \%$ and a false positive rate of 3 to $5 \%$ should be anticipated.

\section{Clinical application of linkage \\ J H EDWARDS \\ Genetics Laboratory, Biochemistry Department, University of Oxford, Oxford.}

The classical application of linkage is the use of the CDE model of the rhesus locus to define paternal heterozygosity at the D locus, assuming no mutation and no crossing error. The use of the loci defined by probes allows similar inferences, but if defined by family studies (bracketing loci or neighbouring loci, as opposed to the flanking loci defined by direct DNA studies), crossing over can lead to both false positives and false negatives. In the presence of a high mutation rate false positives will occur, and it will be formally impossible to diagnose the first carrier by this means. Using $\mathbf{R}$ and $\mathbf{M}$ for recombination and mutation, and small letters for their virtual absence, situations can be classified as $\mathrm{rm}$ (thalassaemia), Rm (accurately diagnosed Huntington's disease in defined communities), and RM (Duchenne). These situations lead to distinct problems.

Seckel syndrome and related disorders E THOMPSON AND M PEMBREY Mothercare Unit of Paediatric Genetics, Institute of Child Health, London.

Seckel syndrome is a rare autosomal recessive syndrome of microcephalic primordial dwarfism. The main diagnostic criteria are intrauterine growth retardation, proportionate short stature, and microcephaly, which are all severe, associated with a 'bird-headed' facial profile and mental retardation. Two children with classical Seckel syndrome are presented who illustrate a number of additional useful diagnostic features. A further sib pair meet sufficient criteria to be classified as Seckel syndrome even though they were taller than previously reported cases. Three children with a similar disorder but with features clearly distinguishing them from Seckel syndrome are discussed. In infancy, the diagnosis of Seckel syndrome may be more difficult since the facial features have not yet fully evolved.

Raised 2 oxoglutarate in the DOOR syndrome M A PATTON, R M WINTER, S KRYWAWYCH, AND M BARAITSER Institute of Child Health, London.

The DOOR syndrome consists of Deafness, Onychodystrophy, Osteolysis, and Retardation. Three further cases are presented and the clinical findings and published reports discussed. A raised level of the organic acid 2 oxoglutarate was found in the plasma and urine in these patients. This is the first time a biochemical abnormality has been described in this syndrome. The relationship between organic acidurias and dysmorphic features is discussed. 